Proximal femoral resection for subluxation or dislocation of the hip in spast...

Steve Ackerly; Coley Vitztum; Brent Rockley; Brad Olney

Developmental Medicine and Child Neurology; Jul 2003; 45, 7; ProQuest Nursing \& Allied Health Source pg. 436

\section{Proximal femoral resection for subluxation or dislocation of the hip in spastic quadriplegia}

Steve Ackerly MD, Resident in Orthopaedic Surgery at the University of Kansas School of Medicine; Coley Vitztum RN MS, Nurse Clinician in Pediatric Orthopedics at Children's Mercy Hospital; Brent Rockley Medical Student at the University of Kansas School of Medicine, Wichita;

Brad Olney* MD, Chief of Orthopedic Surgery, Children's Mercy Hospital, Kansas City, Missouri, USA.

*Correspondence to last autbor at Children's Mercy Hospital, Section of Orthopedic Surgery, 2401 Gilhan Road, Kansas City, MO 64108, USA.

Management of a painful or contracted hip dislocation in individuals with severe spastic quadriplegia is difficult. Clinical and radiographic results of 12 proximal femoral resection-interposition operations performed in seven nonambulatory persons (five males, two females; mean age 14 years, 8 months; age range 6 years 11 months to 19 years 8 months) with severe spasticity were reviewed to determine if pain relief and restoration of motion were maintained. At a mean follow-up of 7 years " months (median 9 years 6 months) all participants maintained a good sitting position and a functional range of motion with improved hygiene. Hip pain was improved in all participants compared with their preoperative status. Proximal femur migration occurred causing slight pain in one person. Heterotopic ossification was observed but was not clinically significant. Complications included traction pin loosening and infection and a late supracondylar femur fracture 3 months after the operation. Proximal femoral resection effectively decreased pain and restored hip motion in those with severe spastic quadriplegia leading to improved sitting and perineal care.
The exact incidence of hip dislocation in cerebral palsy (CP) is unknown, although the majority of hip pathology occurs in persons with the spastic quadriplegic type of CP. Baker and coworkers (1962) analyzed hip pathology in 129 patients (258 hips) with $\mathrm{CP}$ and found that 42 hips (16\%) were subluxated and $31(\mathbf{1 2} \%)$ were dislocated. In the study by Baker and colleagues the participants were not broken down into the type of CP. Howard and colleagues (1985) cvaluated the hips in 85 patients with spastic quadriplegia, and found $10.5 \%$ were dislocated, $10.5 \%$ were subluxated, and $18 \%$ had a dysplastic acetabulum. Lonstein and Beck (1986) found the percentage of hip subluxation or dislocation associated with $\mathrm{CP}$ increased from $7 \%$ for independent ambulators to $60 \%$ for dependent sitters. Treatment of patients with spastic quadriplegia, severely contracted or dislocated hips, and degenerative changes is a challenge. Treatment usually occurs in younger children with severe spasticity or older children with long-standing hip dislocation or subluxation. Not all children with spastic dislocation of the hip experience pain. The incidence of pain associated with hip dislocation or subluxations varies between 33 and $70 \%$, depending on the study. Other problems that may be associated with dislocated or contracted hips include decreased sitting tolerance, skin breakdown, difficulty with nursing care, and problems with perineal hygiene (Bleck 1971, Samilson et al. 1972, Cadman et al. 1978, Rang et al. 1981).

It is generally believed that prevention or early relocation of hip dislocation/subluxation is the treatment of choice in most patients with CP. Early treatment has been described in the literature and includes closed reduction and casting, softtissue releases, open reduction with femoral and/or pelvic osteotomies, and osteotomies without open reduction (Bleck 1980, Wheeler et al. 1984, Silver et al.1985, Hoffer 1986, Lyne et al.1988). These procedures may be helpful in younger patients but may not be appropriate for older children with a long-standing dislocation or severe contractures (Samilson et al.1972, Reimers 1973, Silver et al.1985, Molloy 1986). Hip reconstruction in these patients usually requires some type of salvage procedure because of the contractures and sccondary degenerative changes. Several types of salvage procedures have been described for this type of patient including femoral abcluction osteotomy, hip fusion, hip arthroplasty, and proximal femoral resection-interposition arthroplasty (Parr et al. 1971, Koffman 1981, Root 1982, Kalen et al. 1984, Baxter et al. 1986, Molloy 1986, Root et al. 1986, McCarthy et al. 1988, McHale et al. 1990, Perlmutter et al. 1993, Widmann et al. 1999).

\section{Method}

Between 1988 and 1995, 14 proximal femoral resection interposition arthroplasties were performed in eight non-ambulatory patients with severe spasticity at the University of Kansas Medical Center, Kansas City, USA by the senior author. Only patients with more than 2 years of follow-up were included. One patient with total-involvement CP died 4 months postoperatively (unrelated to the hip procedure), leaving 12 hips in seven patients (five males, two females) for review in this study. The Ethics Committee of the University of Kansas gave approval for the study and informed consent was obtained from the legal guardian of all patients before surgery.

Five patients had total involvement $\mathrm{CP}$, one had hydrocephalus with spastic quadriplegia, and one patient had spina bifida cystica with spastic quadriplegia secondary to a Chiari malformation and hydrocephalus. This last patient also had a 
syringomyelia with thoracic level paraplegia. Mean age at the time of surgery was 14 years 8 months; age range 6 years 11 months to 19 years 8 months.

Preoperatively, 10 hips (six patients) were painful and two hips (one patient with a syringomyelia) were not painful. Five hips were dislocated, three were subluxated, and four had severe hip contractures. The hip contractures included three patients with extension contractures and one patient with an abduction-external rotation contracture. All of the hip contractures made sitting extremely difficult or impossible (see Fig. 1). Three patients (five hips) were unable to sit, three patients (six hips) were able to sit for less than 1 hour, and one patient (one hip) was able to sit for 1 to 3 hours. Perineal care and hygiene were compromised in all patients. One patient with scoliosis and bilateral hip extension contrac- tures had bilateral trochanteric decubiti.

Four patients (seven hips) had prior soft-tissue procedures including adductor, tensor fascia, iliopsoas, and hamstring releases (Table I). One patient had prior bilateral femoral head resections. This involved only resection of the femoral heads, which did not relieve the patient's pain or hip extension contracture. Six patients had scoliosis with two patients having prior spinal instrumentation and fusion.

The operative technique of Castle and Schneider (1978) was employed. The proximal femur was exposed through a lateral approach, extraperiosteally dissected, and resected below the level of the lesser trochanter (see Fig. 2). Extraperiosteal dissection was performed to try and decrease the chance of ectopic bone formation although it is not known if this is necessary. The acetabulum was closed with oversewing of the

Table I: Patient data

\begin{tabular}{lrrrr}
\hline Patient $^{a}$ Age at surgery, $y: m$ & Diagnosis & Side & Priororthopedic procedures \\
\hline 1 & $15: 0$ & Hydrocephalus & Bilateral & $\begin{array}{r}\text { Bilateral adductor releases } \\
\text { left hamstring release } \\
\text { right tensor fascia release }\end{array}$ \\
2 & $14: 10$ & CP & Bilateral & None \\
3 & $19: 10$ & CP & Right & None \\
4 & $11: 7$ & CP & Right & $\begin{array}{r}\text { Left psoas and adductor release } \\
\text { right tensor fascia release } \\
\text { right adductor release } \\
\text { posterior spinal fusion }\end{array}$ \\
5 & $19: 8$ & Spinabifida & $\begin{array}{r}\text { Bilateral } \\
\text { with spastic }\end{array}$ & $\begin{array}{r}\text { Bilateral adductor releases } \\
\text { bilateral hamstring releases } \\
\text { bilateral Achilles lengthening }\end{array}$ \\
6 & & quadriplegia & Bilateral & Bilateral femoral head resections \\
7 & $6: 11$ & CP & Bilateral & Bilateral adductor releases \\
\hline
\end{tabular}

${ }^{a}$ All patients were male except patient 3 .

Figure 1:

Radiograph of 14year-old patient with severe spastic quadriplegia (patient 2). Pelvic obliquity is secondary to fixed contractures which significantly interfered with patient's ability to maintain a seated position.

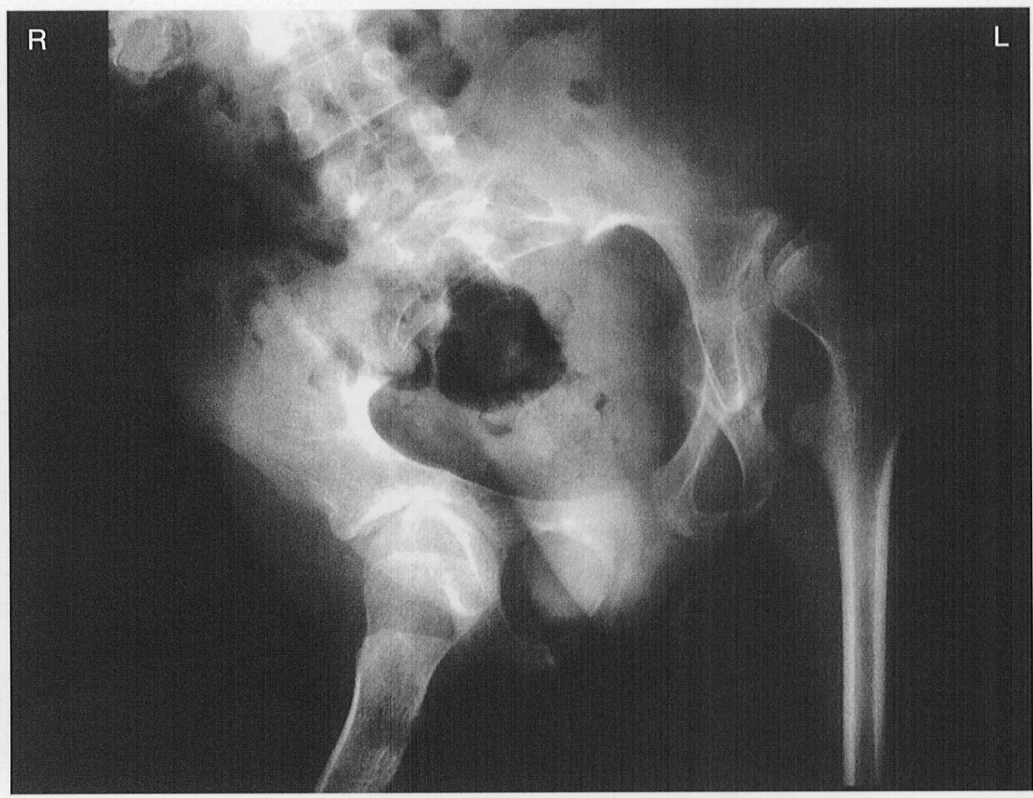


capsule and the vastus lateralis was closed over the end of the proximal femur providing soft tissue interposition. The tensor fascia lata was repaired and the wound closed over drains. Two patients (four hips) had concomitant soft tissue procedures including hamstring releases in four limbs and adductor releases in two limbs. Hamstring releases were performed if knee flexion contractures did not allow postoperative traction after the proximal femoral resection. Postoperatively, six patients were treated in traction for 4 to 6 weeks and one patient was treated in a spica cast because social reasons did not allow for in-hospital traction. 'Traction involved the placement of a distal tibial traction pin with 2.2 to $4.5 \mathrm{~kg}$ of traction. It is believed that a period of postoperative casting or traction is necessary for patient comfort and to allow for soft tissue healing. Physical therapy for hip and lower extremity range of motion (ROM) was instituted in the immediate postoperative period for those patients in traction. External beam irradiation was delivered to the operative field in the immediate postoperative period to try and prevent heterotopic ossification. Six patients received a single dose of irradiation ( 6 to $8 \mathrm{~Gy}$ ) on postoperative days 2 to 5 . The seventh patient received a total of $10 \mathrm{~Gy}$ in four $2.5 \mathrm{~Gy}$ fractions over postoperative days 5 to 8 .

The study design included chart review, examination, and radiographic (anteroposterior pelvis radiograph) review. One patient included in the study died $4 \frac{1}{2}$ years after the procedure, but had adequate clinical and radiographic records for review. Patients were examined for assessment of hip motion, soft tissue integrity, seating position, and tolerance. All patients alive at the time of the study were evaluated by the senior author. Radiographs were reviewed for proximal migration of the femoral shaft and evidence of heterotopic ossification. Heterotopic ossification was classified according to the method described by McCarthy and colleagues (1988). Type I ectopic bone was confined to the top of the femoral shaft and confined within the vastus lateralis. Type II ectopic bone projected from the shaft, extending beyond the vastus lateralis, toward the acetabulum (see Fig. 3). Type III was a pattern of diffuse myositis ossificans distributed between the acetabu- lum and the femur.

The parents or caregivers completed a questionnaire on each patient regarding issues concerning pain, function, sitting tolerance, and hygiene. The caregivers rated each of these issues before and after surgery at the last follow-up. Particular interest was given to lasting improvements in sitting tolerance and hygiene care as well as a subjective decrease in pain. Mean follow-up was 7 years 7 months (median 9 years 6 months), with a range of 2 years 11 months to 9 years 9 months.

\section{Results}

In the clinical evaluation at last follow-up, six of seven patients maintained a functional ROM with near full extension to $90^{\circ}$ of flexion and at least $30^{\circ}$ of abduction. The remaining patient had bilateral resections for anterior hip dislocations and extension contractures. Radiographs in this patient showed significant proximal migration of the femora and there was subsequent development of flexion contractures of 40 and $55^{\circ}$. This patient was still able to achieve a good sitting posture even with the flexion contractures. All patients were able to sit for extended periods of time (at least 4 to 5 hours) following the procedure. Parents and caregivers also noted greatly improved hygiene and perineal care. The time to achieve maximum benefit according to the caregiver questionnaire was 6 to 12 months (range 3 to 18 months). Six of the seven respondents gave the highest rating to each parameter on the questionnaire. They noted greatly improved function and were very satisfied with the surgical outcome. The other respondent gave the second highest subjective rating for each parameter, but was still satisfied with the outcome.

Six of seven patients had hip pain before the proximal femoral resection, five of whom had severe debilitating pain. At the latest follow-up, five of seven patients (nine hips) had no pain and two patients (three hips) had slight pain that did not require medication or compromise their activity.

No patient has required subsequent hip surgery, although two patients have required spinal surgery for scoliosis. There were no problems with wound healing, and only one patient

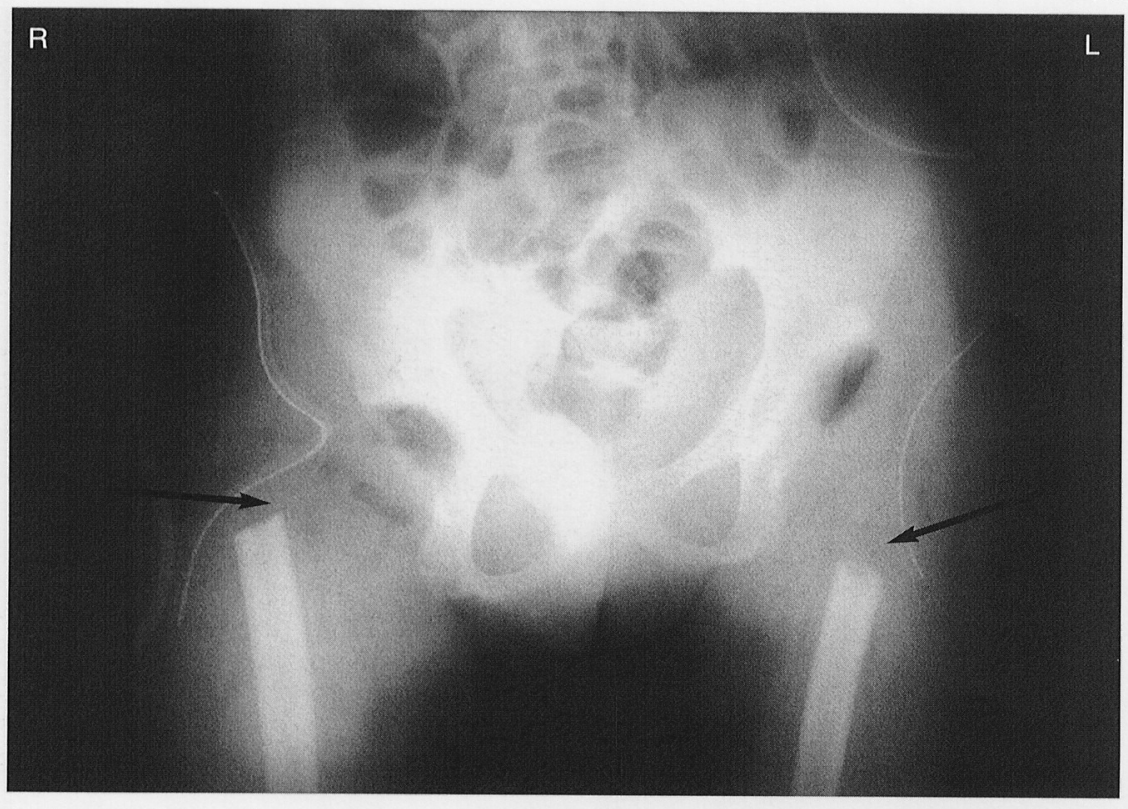

Figure 2: Radiograph of patient 2 obtained immediately postoperatively showing bilateral proximal femoral resections (arrows). 
required a postoperative blood transfusion. Postoperative complications in the first 6 weeks included: cellulitis around an IV site in one patient, pin tract infection in one patient, loosening of the traction pin in four patients, and a small superficial area of skin necrosis due to cast pressure in the patient treated with a postoperative spica cast. Loosening of the traction pin was a common problem because of osteopenic bone and required early removal of the pin in some patients. There were two late complications in the study: the individual with spina bifida developed ischial decubiti that responded to local wound care and one patient with CP sustained a non-displaced supracondylar femur fracture 3 months postoperatively that healed with closed treatment. The development of an ulcer in the patient with spina bifida raises the point that this procedure should be used cautiously in those with altered sensation because it decreases the bony support of the thigh.

Radiographically, all patients developed some degree of heterotopic bone formation. Seven hips demonstrated type I ossification with bone confined to the top of the femoral shaft. One hip exhibited type II changes with a spike-like projection, and four hips had more diffuse type III ossification. In no participant did the heterotopic ossification seem to interfere with the clinical outcome. Even though the resections were preformed extraperiosteally with soft tissue interposition, all hips demonstrated some degree of proximal femoral migration. The migration was graded based on the level of the proximal femur in relation to the acetabulum: grade I, inferior to the acetabulum; grade II, at the level of the acetabulum; and grade III, superior to the acetabulum. Using this grading system four hips were grade I, three hips were grade II, and five hips were grade III. Of the grade-III hips, one patient with bilateral involvement had only slight pain, while the remaining three hips were not painful.

\section{Discussion}

The optimal treatment of non-ambulatory CP patients with painful hip dislocation remains controversial. Open reduction with a femoral and pelvic osteotomy seems to work well in younger patients with a recently dislocated hip, without signifi- cant contractures or degenerative changes. Older patients with long-standing hip dislocations or patients with significant contractures or degenerative changes are usually only candidates for salvage procedures. Not all these patients require surgical treatment; the primary indications for treatment are pain or deformity that significantly interfere with the patient's general care or positioning. Salvage procedures for this difficult problem have included hip arthrodesis, abduction osteotomy, total hip arthroplasty, and proximal femoral resection.

Root and colleagues (1986) reported good results in seven of eight patients with hip arthrodesis. These were older patients with unilateral hip problems, half of whom were ambulatory. There was a high complication rate with this procedure, and the authors only recommended an arthrodesis in younger patients with unilateral hip disease, a normal contralateral hip, and an uninvolved spine. Root and colleagues (1986) and Koffman (1981) have reviewed their results of total hip arthroplasty for painful dislocated hips in patients with CP. The procedure is more technically demanding and revision rates are higher in this population. Pain is relieved and function improved, although the results appear to be better in older $C P$ patients with degenerative joint disease of the hip who are able to stand or walk.

Gabos and coworkers (1999) evaluated 11 non-ambulatory patients with severe $C P$ and painful degenerative arthritis in 14 hips. The authors treated the patients with proximal femoral resections but used humeral implants as a prosthetic interposition arthroplasty. At the time of surgery attempts were made to keep the prosthesis reduced in the true or false acetabulum. Glenoid components were used in some patients. Four of the 14 hips dislocated within 4 months of the procedure, but 10 of 11 patients had good pain relief with this procedure.

McHale and coworkers (1990) reported results of subtrochanteric valgus osteotomy with femoral head resection for painful spastic dislocated hips in individuals with CP. At shorter follow-up (mean $2^{1 / 2}$ to 3 years) results appear similar to proximal femoral resection in terms of functional outcome and rates of complications. Proximal femur migration did not
Figure 3: Radiograph of patient 2 obtained 3 years postoperatively. This patient's caregiver reported no pain at this time and unlimited ability to maintain a seated position. There is evidence of type II beterotopic ossification near end of eachfemur (arrow).

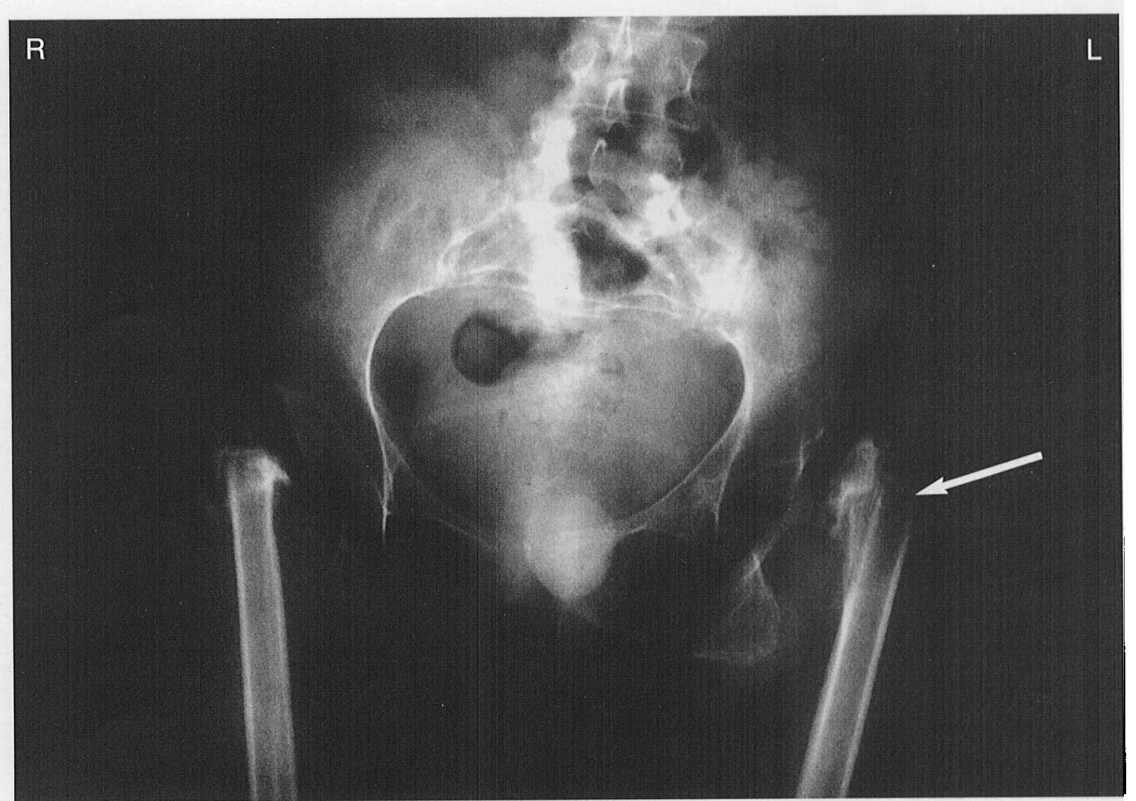


occur in the McHale study, although two of five patients had residual pain that may have been related to continued bony contact between the femoral neck and the acetabulum within the closed capsule. In each of these studies one patient had problems related to the hardware used to stabilize the valgus osteotomy.

Perlmutter and colleagues (1993) reported results from a retrospective study regarding proximal femoral resection in the management of the painfully dislocated or subluxated hips of older children with severe disabilities as the result of spastic hip disease. At follow-up six of the 13 patients required prescription medications for pain. The presence of continued bony contact between the femur and pelvis correlated well with the postoperative pain level and the quantity of proximal femoral resection. The six patients continued to show bony contact either by heterotopic ossification or by continued proximal femoral impingement onto the pelvis. The authors of the study recommended using a modified Castle technique which was further outlined in a study carried out by McCarthy. This technique describes extraperiosteal dissection with greater resection of the proximal femur to include $3 \mathrm{~cm}$ of bone below the level of the lesser trochanter. The authors also endorsed the use of traction for 3 to 6 weeks to prevent early proximal migration of the femur postoperatively.

Widman and colleagues (1999) recently conducted an outcome study of proximal femoral resection in 13 patients (18 hips) with an average age of 26 years 7 months. Five of the hips were treated with single-dose radiation therapy postoperatively in an attempt to lower the recurrence of heterotopic ossification. 'The results of the radiation therapy showed a statistically significant decrease in the incidence and severity of heterotopic ossification in the five hips that received radiation compared with the 13 hips that received no radiation therapy. Proximal femoral resection resulted in a decrease in pain and an increase in sitting tolerance in all 13 patients. Maximal pain relief for patients in this study was achieved at an average of 5.6 months postoperatively.

\section{Conclusion}

In our study at last follow-up, functional improvements were reported in all patients who underwent proximal femoral resection for painful hip dislocation or contracture secondary to spastic quadriplegia. The results included: improved ROM, better tolerance of a sitting position, and improved perineal hygiene. In addition, pain experienced by patients postoperatively was dramatically reduced compared with preoperative levels. Despite postoperative irradiation, heterotopic bone ossification was observed radiographically in each patient. However, in no patient did the degree of heterotopic ossification interfere with clinical outcome such as continued pain or hip stiffness. Besides one occurrence of proximal femoral migration, complications following proximal femoral resection were relatively minor and consisted of traction pin loosening, one pin infection, and one superficial skin lesion. No surgical revision has been necessary for any patient. Proximal femoral resection is an effective salvage procedure for reducing pain and restoring hip motion in patients with severe spastic quadriplegia and painful dislocated or contracted hips.

DOI: $10.1017 / \mathrm{S} 0012162203000823$
Accepted for publication 17th December 2002

\section{References}

Baker LD, Dodelin R, Bassett FH. (1962) Pathologic changes in the hip in cerebral palsy: incidence, pathogenesis, and treatment. $J$ Bone Joint Surg 44A: 1331-42.

Baxter M, D'Astrous J. (1986) Proximal femoral resection interposition arthroplasty: salvage hip surgery for the severely disabled child with CP. J Pediatr Orthop 6: 681-5.

Bleck EE. (1971) Hip deformities in cerebral palsy. Instr Course Lect $20: 54-82$.

Bleck EE. (1980) The hip in cerebral palsy. Ortbop Clin North Am 11: 79-104.

Cadman D, Richards J, Feldman W. (1978) Gastroesophageal reflux in severely retarded children. Dev Med Child Neurol 20:95-8.

Castle M, Schneider C. (1978) Proximal femoral resection interposition arthroplasty. JBone Joint Surg 60A: 1051-4.

Gabos PG, Miller F, Galban MA, Gupta GG, Dabney K. (1999) Prosethetic interposition arthroplasty for the palliative treatment of end-stage spastic hip disease in nonambulatory patients with cerebral palsy. J Pediatr Orthop 19: 796-804.

Hoffer M. (1986) Current concepts review: management of the hip in cerebral palsy.J Bone Joint Surg 68A: 629-31.

Howard DB, McKibbin B, Williams LA, Mackie I. (1985) Factors affecting the incidence of hip dislocation in cerebral palsy. $J$ Bone Joint Surgery 6\% $\mathbf{6}$ : $530-2$.

Kalen V, Gamble J. (1984) Resection arthroplasty of the hip in paralytic dislocations. Dev Med Child Neurol 26: 341-6.

Koffman M. (1981) Proximal femoral resection or total hip replacement in severely disabled cerebral-spastic patients. Orthop Clin North Am 12:91-100.

Lyne ED, Katcherian DA. (1988) Slotted acetabular augmentation in patients with neuromuscular disorders. J Pediatr Orthop 8: 278-84.

Lonstein J, Beck K. (1986) Hip dislocation and subluxation in cerebral palsy.J Pediatr Orthop 6:521-6.

McCarthy R, Simon S, Douglas B, Zawacki R, Reese N. (1988) Proximal femoral resection to allow adults who have severe cerebral palsy to sit.J Bone Joint Surg "0A: 1011-16.

McHale K, Bagg M, Nason S. (1990) Treatment of the chronically dislocated hip in adolescents with cerebral palsy with femoral head resection and subtochanteric valgus osteotomy. J Pediatr Orthop 10: 504-9.

Molloy M. (1986) The unstable paralytic hip: treatment by combined pelvic and femoral osteotomy and transiliac psoas transfer./ Pediatr Orthop) 6: 533-8.

Parr P, Croft C, Enneking W. (1971) Resection of the head and neck of the femur with and without angulation osteotomy. J Bone Joint Surg 53A: 935-8.

Perlmutter M, Synder M, Miller F, Bisbal R. (1993) Proximal femoral resection for older children with spastic hip disease. Dev Med Child Neurol 35: 525-31.

Rang M, Douglas G, Bennett G, Koreska J. (1981) Scating for children with cerebral palsy.J Pediatr Orthop 1: 279-87.

Reimers J. (1973) Static and dynamic problems in spastic cercbral palsy.J Bone Joint Surg 55B: $822-7$.

Root L. (1982) Total hip replacement in young people with neurological disease. Dev Med Child Neurol 24: 186-8.

Root I, Goss J, Mendes J. (1986) The treatment of the painful hip in cerebral palsy by total hip replacement or hip arthrodesis.J Bone and Joing Surg 68A: 590-7.

Samilson R, Tsou P, Aamoth G, Green W. (1972) Dislocation and subluxation of the hip in cerebral palsy.J Bone Joint Surg 54A: 863-73.

Selva G, Miller F, dabney K. (1998) Anterior hip dislocation in children with cerebral palsy.J Pediatr Orthop 18: 54-61.

Silver R, Rang M, Chan J, de la Garza J. (1985) Adductor release in nonambulant children with cerebral palsy.J pediatr Orthop 5: 672-7.

Wheeler M, Weinstein S. (1984) Adductor tenotomy-obturator neurectomy.J Pediatr Ortbop 4: 48-51.

Widmann R, Do 'T, Doyle S, Burke S, Root L. (1999) Resection arthroplasty of the hip for patients with cerebral palsy: an outcome study.JPediatr Orthop 19:805-10. 